\title{
Spital im Bild
}

Erhard Taverna

Ein tamilischer Tellerwäscher zeigt mir den Weg zum Grafikcenter, wo ich im Fotostudio verabredet bin. Hier im Untergeschoss arbeiten die Leute vom Kommunikationsdienst, die Hausdrucker und Grafiker, die Beschrifter und die Hersteller der Personalausweise. Eine flache, weisse Bühne und Scheinwerfer auf hohen Stativen markieren das «Studio». Die beiden Fotografinnen sind Profis, die zuvor Fotoreportagen und Porträts gemacht und für Mode, Industrie und Werbung

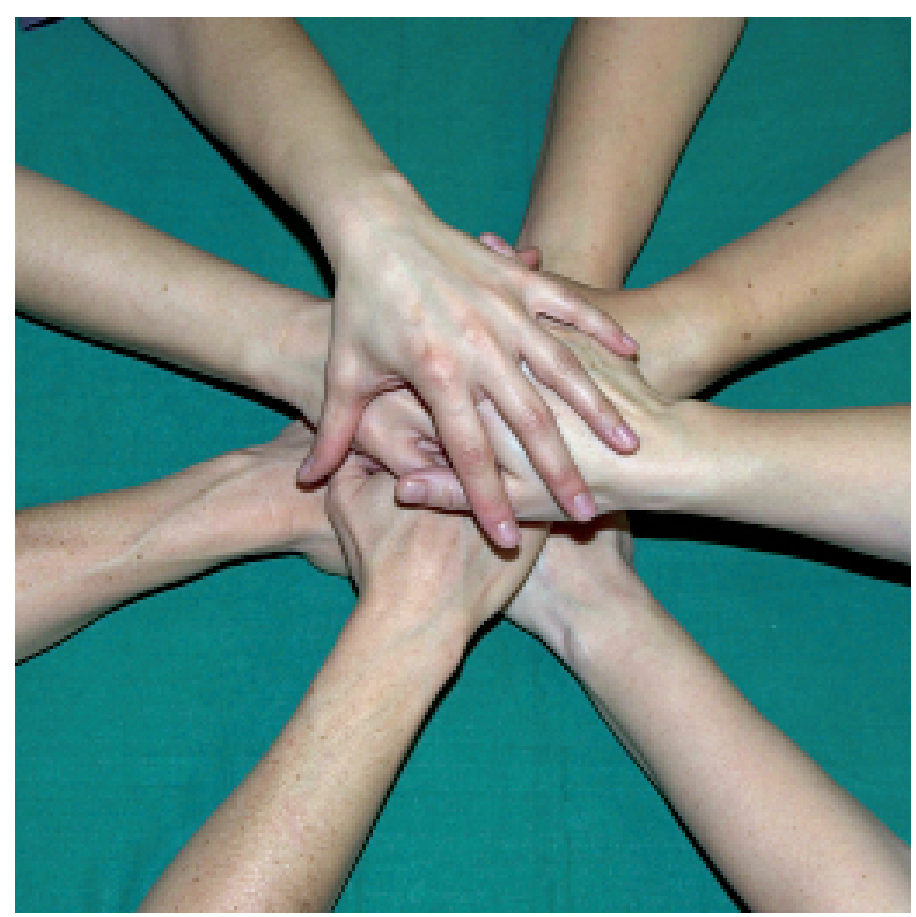

rückgegangen. Nur in seltenen Ausnahmen betreten die Fotografinnen einen OP und überhaupt nie die Augenklinik, wo spezialisierte Fotografen beschäftigt werden.

Die breite medizinische Nutzanwendung der Fotografie begann 1851 mit der Entwicklung des nassen Kollodiumverfahrens. Die alten Bildersammlungen jener Pionierzeiten sind heute gefragte museale Prunkstücke, Kulturzeugnisse und Meilensteine der Entwicklung. Doch Ziel und Zweck sind seit derersten Daguerreotypie in der Klinik gleichgeblieben. Die vielen neuen bildgebenden Verfahren haben aber mit der Digitalisierung nicht nur den Film, sondern auch den gelernten Fotografen aus dem engeren medizinischen Bereich verdrängt. Spezialisten sind noch gefragt, wo es um Spezialeffekte, Veröffentlichungen, um Lehrzwecke und Vorträge geht. Oder wenn sie während unzähliger Arbeitsstunden alte Diasammlungen und Röntgenfilme reinigen, einscannen, auf einem Bildschirm bearbeiten und für das neue Pixelarchiv digitalisieren müssen.

Der Sachfotografie gilt heute der grösste Aufwand, etwa mit Abbildungen neuer Instru-

gearbeitet haben. Für die klinische Fotografie gilt «learning by doing» zum Beispiel, wenn mobile Patienten für Aufnahmen gebracht werden. Die standardisierten Fotos dokumentieren einen Befund vor und nach der Operation. Zum Beispiel von Übergewichtigen, mit und ohne Kleidern, für Verlaufskontrollen und Kostengutsprachen für die Krankenkassen, oder vier Belichtungen vor der Mammareduktion für die plastischen Chirurgen, Bilder von Ohren, Nasen und anderen Gesichtsteilen als Planungsgrundlagen und Belege für Korrekturen und Liftings. Die meisten Patienten sind von der HNO, der Orthopädie und Endokrinologie. Wer nicht ins Studio kommt, wird auf der Abteilung besucht. Doch seit die Mediziner ihre digitalen Aufnahmen selber schiessen, sind die Aufträge massiv zu- mente für Kataloge und Gebrauchsanweisungen und für Instruktionen wie Händedesinfektion, Apparatekenntnisse und Verbandtechniken. Der gewaltige Papierausstoss aus der hauseigenen Druckerei gehört zum Nervensystem des Grossspitals. Zahlreiche Interessenten setzen sich ins Bild: Die Fachschulen informieren mit einem Faltblatt über die Ausbildung der Pflegefachfrau oder der Fachangestellten Gesundheit, das neue Muskelzentrum/ALS clinic macht sein Team bekannt, Tausende von Patientenbroschüren beruhigen allein schon durch ihre visuelle Qualität, die Infowand in der Cafeteria erinnert an den Tennisplausch, an den nächsten Aktionstag, an eine Lehrveranstaltung oder das neue Telefonverzeichnis. Diplomierte, Prüfungsabgänger, neue Chefärzte, Eltern und Podiumsteilnehmer, sie 
alle werden zum mehr oder weniger fotogenen Bestandteil der täglichen Bilderflut; alle wollen durch ihren Auftritt überzeugen. Das neue Personal wird vorgestellt, das alte Kader verabschiedet, die Radioonkologie inszeniert das modernste Bestrahlungsgerät. Neue Dienstleistungen informieren, werben und konkurrenzieren wiederum andere Stellen, die mit Text und Bild ihre unverzichtbaren Angebote verbreiten. Die Fotografie ist das Schmiermittel im Getriebe der hochkomplexen Spitalmaschinerie, denn alle Abteilungen verlangen unersättlich Bilder. Wo Informatik und Kommunikation für die Werbung nach innen und aussen sorgen, ist die professionelle Fotografie unentbehrlich. Dazu gehört auch, dass die Geschäftsleitung viermal jährlich ein Mitarbeitermagazin für ihre Angestellten vertreibt. In der perfekt gestalteten Hauszeitung erfährt jeder, was die anderen tun, hat jeder einmal seinen Auftritt, sorgen Bildberichte für ein Wir-Gefühl. Die Spitalführung setzt die unternehmerischen Schwerpunkte, die Fotoreportage vom Personalfest zeigt denen, die nicht dabei waren, wie toll und locker die Stimmung war.
Gegen aussen verströmen die Jahresberichte Optimismus und Vertrauen. Sie zeigen uns seriöse Verwaltungsräte und Direktoren in tadellosen Anzügen mit bunten Krawatten, die kompetent für die Maximierung unserer Steuergelder sorgen. Wir freuen uns an lächelnden Patienten in dezent moderner Bettwäsche, am blitzsauberen Laborgerät, am trendig gestylten Verwaltungspersonal, am lässigen Overall des Betriebstechnikers und an der frisch gewaschenen Ambulanz.

Wie sich ihre Stellung in den letzten Jahren entwickelt habe, möchte ich zum Schluss von der Fotografin wissen. Mit dem technischen Innovationsdruck habe sich die Konkurrenz verstärkt und die Wertschätzung vermindert. Stellenstreichungen haben das Team reduziert. Aber sie seien schon immer Einzelkämpferinnen und Exotinnen gewesen. Man müsse sich eben in Erinnerung rufen, «zeigen, dass es uns gibt.» Es wird sie weiter geben, denn ohne sie wären wir nie im Bild. 